EPJ Web of Conferences 45, 01059 (2013)

DOI: $10.1051 /$ epjconf/20134501059

(C) Owned by the authors, published by EDP Sciences, 2013

\title{
CFD comparison with centrifugal compressor measurements on a wide operating range
}

\author{
P. Le Sausse ${ }^{1, a}$, P. Fabrie ${ }^{1}$, D. Arnou ${ }^{2}$, and F. Clunet ${ }^{2}$ \\ 1 Université de Bordeaux, IPB, UMR5251, ENSEIRB-MATMECA, Talence, France \\ 2 Johnson Controls Industries, France
}

\begin{abstract}
Centrifugal compressors are widely used in industrial applications thanks to their high efficiency. They are able to provide a wide operating range before reaching the flow barrier or surge limits. Performances and range are described by compressor maps obtained experimentally. After a description of performance test rig, this article compares measured centrifugal compressor performances with computational fluid dynamics results. These computations are performed at steady conditions with R134a refrigerant as fluid. Navier-Stokes equations, coupled with k- $\varepsilon$ turbulence model, are solved by the commercial software ANSYS-CFX by means of volume finite method. Input conditions are varied in order to calculate several speed lines. Theoretical isentropic efficiency and theoretical surge line are finally compared to experimental data.
\end{abstract}

\section{Introduction}

Turbocompressors are widely used in industrial applications. One type of these machines is the centrifugal compressor, which consists of converting kinetic energy in pressure energy.

Turbocompressors performances are to used to be analysed by two main result data : pressure ratio and efficiency. These two parameters are varying with volume flow and with the machine rotational speed.

The compressor designer objective could be to obtain wider performance map with higher pressure ratio while improving efficiency. The oldest and most accurate method to validate a new geometry is to make a benchmark on a test rig. However, this method involves a substantial time and money expending.

Since some years, computational flow methods (CFD) are developing to assess compressor performances. It enables to gain many time between the concept and the test. Moreover, several shapes can be compared quickly without spending money in materials and manufacturing. However, computations may last for a very long time without the certainty to have a precise result.

That is why this article is focused on the comparison of these two testing methods.

First literature we can find about this subject appeared in the nineties [1] with computation on only one operating point, which is near peak-efficiency. On this kind of studies [2], comparison are centered on flow fields and angles, sometimes with a transient component [3]. Comparison between experimental and CFD is a good way to improve mathematical formula as turbulence model [4]. It is also shown that turbulence models accuracy is more or less variable in function of the operating conditions [5].

During the ten last years, computer improvements enable

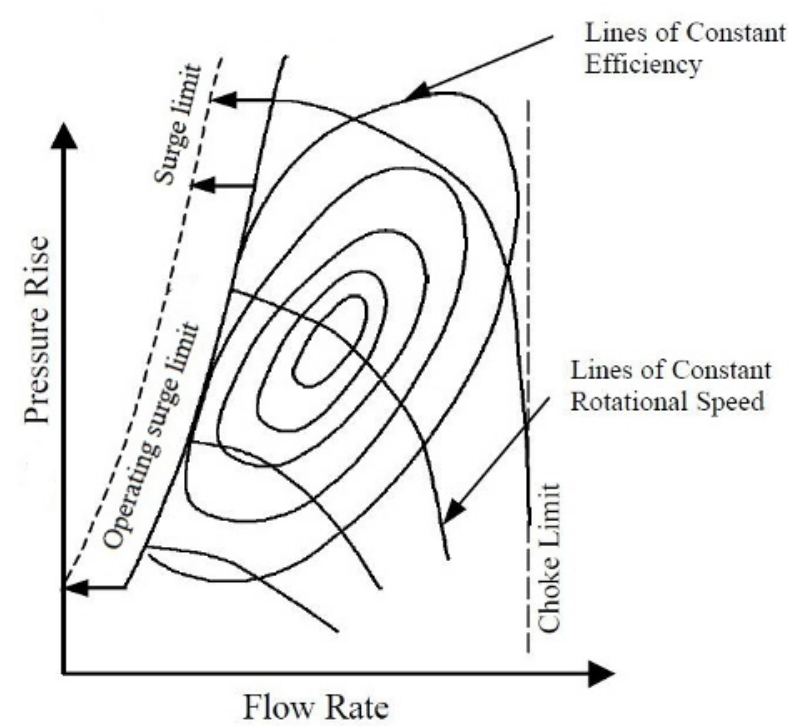

Fig. 1. Compressor performance map

to multiply operating points computations. Then comparisons between CFD and experimental tests are possible on a wide operating range.

Turbocompressors maps are limited by two flow phenomena :

- the choke limit for high flow rate matches a transonic flow somewhere in the compressor.

- the surge limit is reached at low flow rate and is dependent on the global system. Therefore, this phenomenon is generally more difficult to anticipate.

The figure 1 is a schematic of a typical compressor map within limit curves are shown. The reach of the surge limit may destroy a compressor, that is why a security is required with a operating surge limit.

\footnotetext{
a e-mail: Paul.lesausse@jci.com
} 
Some papers are about one constant rotational speed [6], but most of actual literature allows to differentiate several speed lines and conclude that gap between CFD and experimental increases with speed [7-9]. The latest studies try to assess the surge appearance by CFD [10], and even compute system to move the surge line back [11].

The goal of this study is to tally CFD results with experimental data with a geometry considered as perfectly known. A further application will consist in improve geometry by CFD to get best machine performances as the study [12].

\section{Methodology}

In this section is described the methodology of the experimental setup and, then, the numerical simulation setting up.

\subsection{Experimental rig and testing}

All measures described in this article took place in the company "Johnson Controls Industries" (JCI), specialized in the refrigeration domain. In a chiller, a refrigerant is circulating in a closed loop, passing successively in the evaporator, the centrifugal compressor, the condenser and expansion valve. The chiller is connected to a dedicated test rig, used to adjust its operating conditions.

As it is described on the figure 2, some sensors are located on the frigorigen fluid circuit, and some other take directly the measure on the external fluid. All sensors are calibrated each year and comply with AHRI 551/991.

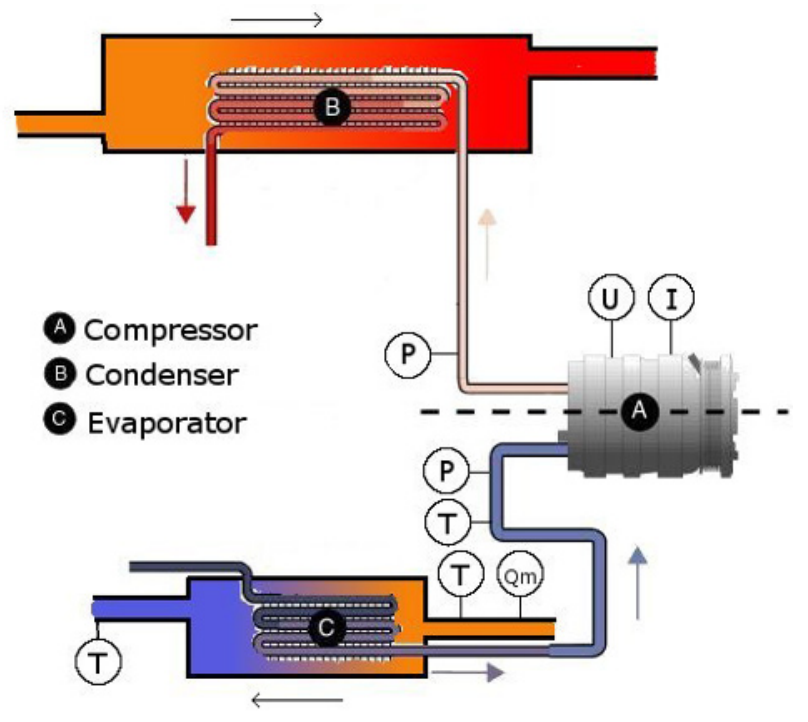

Fig. 2. refrigeration cycle

\subsubsection{Measurement of frigorific power}

Two temperature measurements are done at evaporator inlet and outlet. Each measurement location is composed of four sensors in order to reduce statistic error. Moreover, mass flow is measured with a temporal integration to limit dynamic fluctuation measurements.

Thus, we get frigorific power :

$$
P_{\text {evapo }}=Q_{m} * C p *\left(T_{\text {out }}-T_{\text {in }}\right)
$$

With $Q_{m}$ the mass flow,

$T_{\text {in }}$ the evaporator inlet temperature,

$T_{\text {out }}$ the evaporator outlet temperature.

The accuracy of temperature sensors is $\pm 0.05 \mathrm{~K}$. The mass flow sensor one is $\pm 0.5 \%$.

\subsubsection{Measurement of pressure ratio}

The pressure ratio expression is get by two pressure sensors at compressor suction and discharge :

$$
\tau=\frac{P_{\text {dis }}}{P_{\text {suct }}}
$$

With $P_{d i s}$ the compressor discharge pressure, $P_{\text {suct }}$ the compressor suction pressure.

Precision we get for pressure measurement is about $\pm 0.3 \%$.

\subsubsection{Calculation of compressor isentropic efficiency}

The efficiency is calculated from electric power measure at motor input with an accurate motor losses theoretical model. Motor tension and intensity measurements are performed thanks to the use of three sensors of each type. Precision of these sensors may vary between 0.5 and $1 \%$.

Through fluid tables, and in measuring the suction compressor temperature, we are able to get a suction enthalpy $\left(H_{\text {suct }}=f\left(P_{\text {suct }}, T_{\text {suct }}\right)\right)$ and a suction entropy $\left(S_{\text {suct }}=\right.$ $\left.g\left(P_{\text {suct }}, T_{\text {suct }}\right)\right)$. Then, discharge pressure enables to get discharge isentropic enthalpy $\left(H_{\text {is dis }}=h\left(P_{\text {dis }}, S_{\text {suct }}\right)\right)$. Finally, we get :

$$
\Delta H_{\text {is }}=H_{\text {is dis }}-H_{\text {suct }}
$$

These previous data are sufficient to calculate isentropic efficiency :

$$
\frac{1}{\eta_{i s}}=\frac{P_{\text {motor }}-P_{\text {losses }}}{Q_{m} \Delta H_{i s}}
$$

With $\eta_{i s}$ the isentropic efficiency,

$P_{\text {motor }}$ the electric motor power in W,

$P_{\text {losses }}$ the motor losses power in W. 


\subsection{Numerical settings}

A centrifugal compressor, also called radial compressor, is composed of many components as indicated by the figure 3 :

- the convergent axially guides fluids into the impeller

- the impeller is a rotating part and is the main compressor element because it provides work to the fluid. The blades press the fluid which undergoes a centrifugal acceleration.

- the diffuser increases pressure energy in slowing fluid down.

- the scroll plays the same role as the diffuser and transfers fluid to other process components.

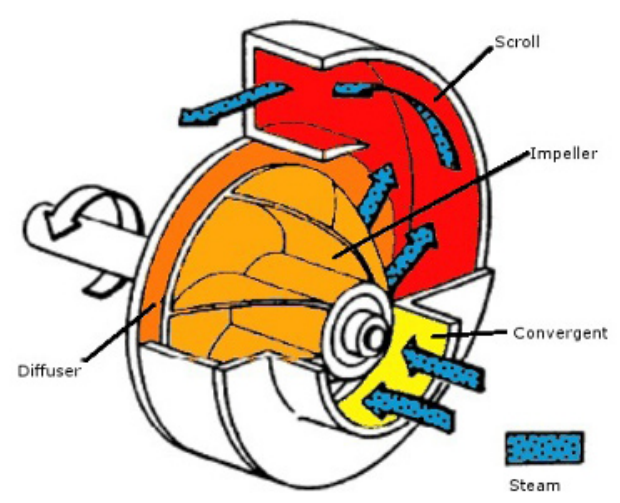

Fig. 3. Compressor components

\subsubsection{Model parameters}

A reference impeller is analysed using the commercial computational fluid [13] dynamic software ANSYS-CFX using a finite volume method [14].

Due to periodicity features of the impeller geometry, only one passage containing one main blade and one splitter (secondary blade) was modelled. To ensure a good transition between impeller and diffuser and to improve computation costs, we apply the same periodicity on diffuser geometry.

The model uses a structured mesh made of hexahedrons in all geometries excepted in the scroll volume composed of tetrahedrons as we can observe it in the figure 4 . The mesh contains several thin layers along wall for accurate boundary layers computation.

The boundary conditions are following:

- total pressure at the inlet

- total temperature at the inlet

- static pressure at the outlet

- $5 \%$ turbulence intensity at the inlet

- adiabatic wall with $8.5 \mu \mathrm{m}$ of roughness on impeller and diffuser

- adiabatic wall with $0.5 \mathrm{~mm}$ of roughness on scroll

Computations have solved steady and compressible Navier-

Stokes equations, coupled with $k-\varepsilon$ turbulence model,

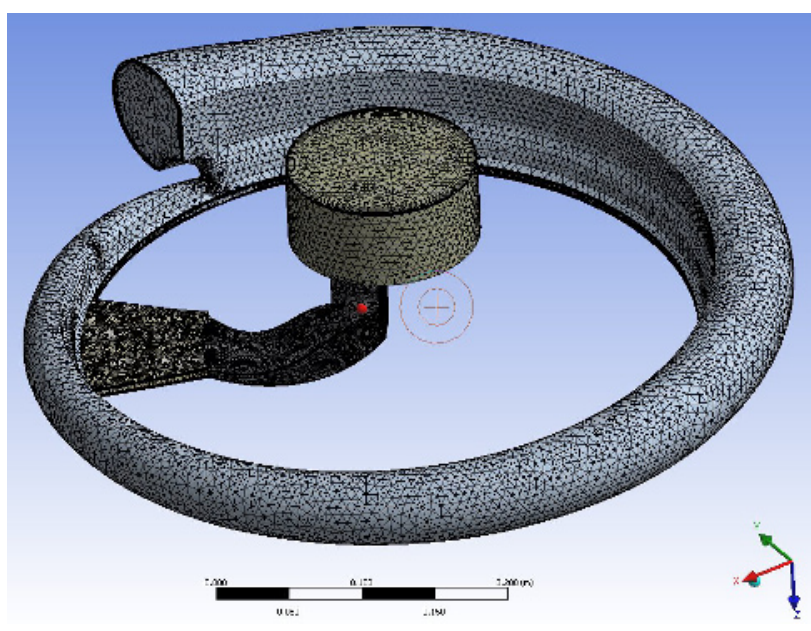

Fig. 4. Mesh model

with R134a as frigorific fluid.

\section{Results and discussion}

Before the comparison between CFD computations and experimental results, the impact of mesh dimension is analysed.

Then, a mesh will be chosen to obtain results on several speed lines.

\subsection{Mesh dimension analysis}

After having calibrated the model a first time, eight mesh dimensions are tested, referred as following:

-Coarse mesh : 126000 elements

-Reference mesh : 153000 elements

-Medium mesh : 297000 elements

-Fine mesh : 427000 elements

-Fine mesh $2: 754000$ elements

-Fine mesh 3:1171000 elements

-Fine mesh $4: 1417000$ elements

-Fine mesh $5: 1686000$ elements

Among these meshes, four impeller meshes are shown on figure 5 .

Note 1 : the scroll elements number remains constant, only periodic features are impacted by mesh modifications. ${ }^{1}$

Note 2: This analysis has been done on a operating point close to the efficiency peak.

The objective of these computations is to observe variables convergence when mesh is refining. As we have indicated in the section 2.2.1, a total pressure is set at the model inlet and a static pressure at the outlet. That is why

\footnotetext{
${ }^{1}$ For comparison, fine mesh 3 (1 171000 elements) would be composed of about 13000000 elements for a model without periodicity.
} 


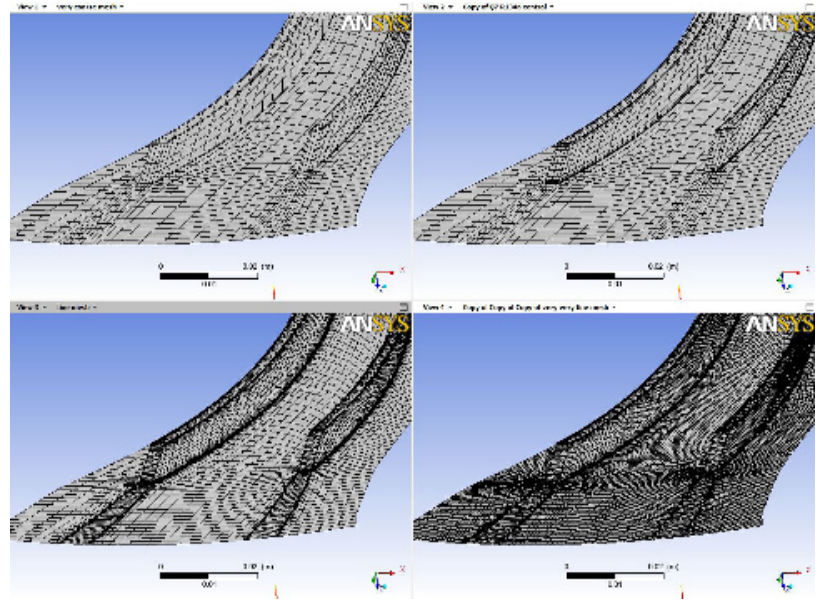

Fig. 5. Impeller element number - top left : coarse mesh ; top right : reference mesh ; bottom left : medium mesh ; bottom right : fine mesh 5

the inlet static pressure may vary, what impact static pressure ratio. The figure 6 shows that this result is converging with mesh refinement.

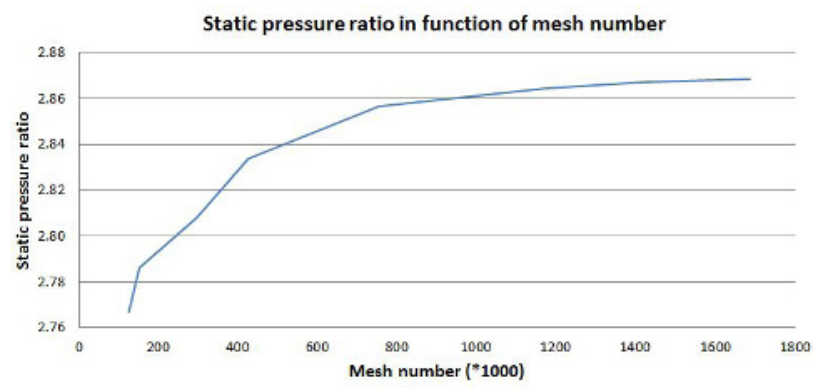

Fig. 6. CFD static pressure ratio in function of element number

Calculations enable to get pressure ratio and mass flow values. Then we are able to compare experimental and CFD rotational speed which is characterized by the variable Mach :

$$
M=\frac{\omega \frac{D}{2}}{a}
$$

With $\omega$ the rotational speed in $\mathrm{rad} / \mathrm{s}$,

$D$ the exit impeller diameter in $\mathrm{m}$,

$a$ the speed of sound compressor inlet in $\mathrm{m} / \mathrm{s}$.

To compare easily experimental and CFD value, we add the parameter $M_{\text {ratio }}=\frac{M_{C F D}}{M_{\text {exp }}}$. The figure 7 demonstrates that the mach ratio converges on a value very close to 1 .

\subsection{Experimental and CFD comparisons}

The purpose of this paragraph is to introduce experimental and CFD results and analyse common and different points. For all computations, a mesh composed of 427000 elements is used. This choice is justified by a good ratio accuracy/computation time. Indeed, previous paragraph values

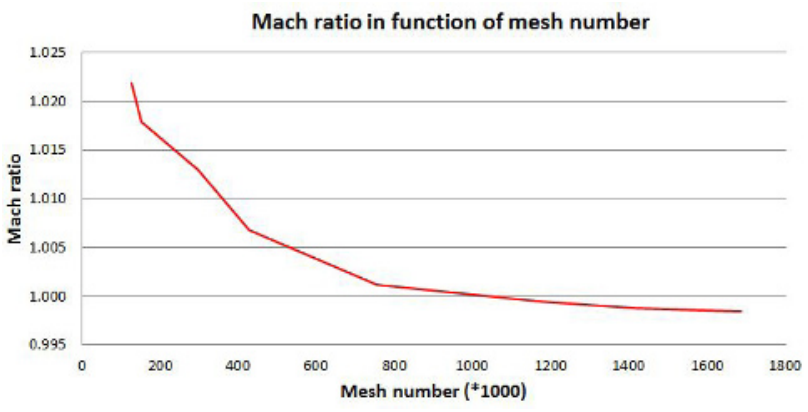

Fig. 7. Mach ratio in function of elements number

an error of about $0.7 \%$ with experimental mach number results.

Some corrections will contribute to offset CFD results.

As we can see on figure 1, speed lines are function of two parameters : flow rate and pressure rise. Several formulations are used to express them. Here we will use Theta for the flow rate ratio and the pressure ratio for load rise as it is already defined in the equation 2 .

We will also describe the isentropic efficiency which is commonly used in the turbomachine domain. The experimental calculation is expressed on the equation 4, CFD calculation is get by :

$$
\eta_{\text {is }}=\frac{\Delta H_{\text {is }}}{\Delta H_{\text {real }}}=\frac{H_{\text {out is }}-H_{\text {in }}}{H_{\text {out }}-H_{\text {in }}}
$$

With $H_{\text {out }}$ is the outlet isentropic enthalpy in $\mathrm{kJ} / \mathrm{kg}$, $P_{\text {in }}$ the compressor inlet pressure in $\mathrm{kJ} / \mathrm{kg}$, $P_{\text {out }}$ the compressor outlet pressure in $\mathrm{kJ} / \mathrm{kg}$.

The efficiency and the rotational speed will be expressed in percentage in relation to a reference variable :

$$
\begin{aligned}
\eta_{\%} & =\frac{\eta_{\text {is }}}{\eta_{\text {ref }}} * 100 \\
M_{\%} & =\frac{M}{M_{\text {ref }}} * 100
\end{aligned}
$$

The use of all these non-dimensional variables enables to compare performances of different compressor geometries or other fluids.

\subsubsection{Uncorrected comparison}

Five speed lines have been calculated from mach number equal to $62 \%$ to $108 \%$, including also $M=67 \%, M=92 \%$ and $\mathrm{M}=100 \%$. Number of points by line is varying.

CFD calculations are diverging when the static pressure set at the outlet of the model is not reachable. In this study, only $\mathrm{M}=100 \%$ line reached the CFD surge limit.

The pressure ratio characteristics from both the CFD calculations and the experiments are shown on the figure 8 . We can note that differences between CFD and experimental results are varying with the impeller rotational speeds. Indeed, higher is the rotational speed, upper is the CFD 




Fig. 8. Uncorrected pressure ratio comparison

line compared with the experimental line.

This phenomenon may be explained by the fact that the CFD model does not included all compressor elements. A compressor is composed of a downstream convergent element and an other upstream divergent one.

That is why calculated pressures at the CFD model bounds are not the same as the real compressor. We may suppose that differences between both CFD and experiment lines are increasing with the dynamic pressure rising. It explains the consequent difference for high velocity speeds.

The figure 9 displays the relation between flow rate and isentropic efficiency for the same values of rotational speeds as previously. Gaps between CFD and experiments are more constant, maximum efficiency values are close to 99\% for experiments against about $101 \%$ for CFD.

However, experimental lines are steeper than CFD curves.

\subsubsection{Correction of the model composition}

The addition of the missing components in the CFD model would involve a higher mesh element number and would increase considerably time computations.

A second solution to correct errors due to this compressor parts lack consists in importing CFD results in a calculation software in which a assessment of the flow is done in downstream and upstream components.

The figure 10 represents computations results to which corrections have been applied. It is interesting to notice an impact specially on high rotational speed line as we supposed in the previous paragraph.

The flow is estimated as a isentropic evolution in downstream and upstream components. However, this kind of

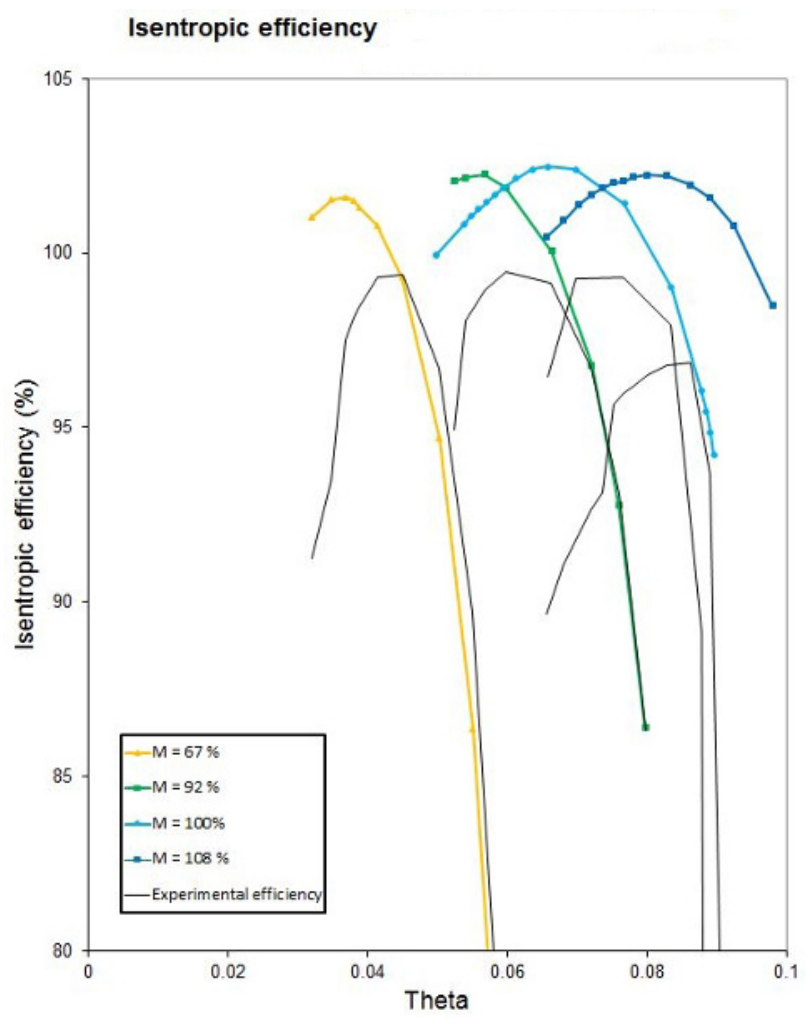

Fig. 9. Uncorrected efficiency comparison

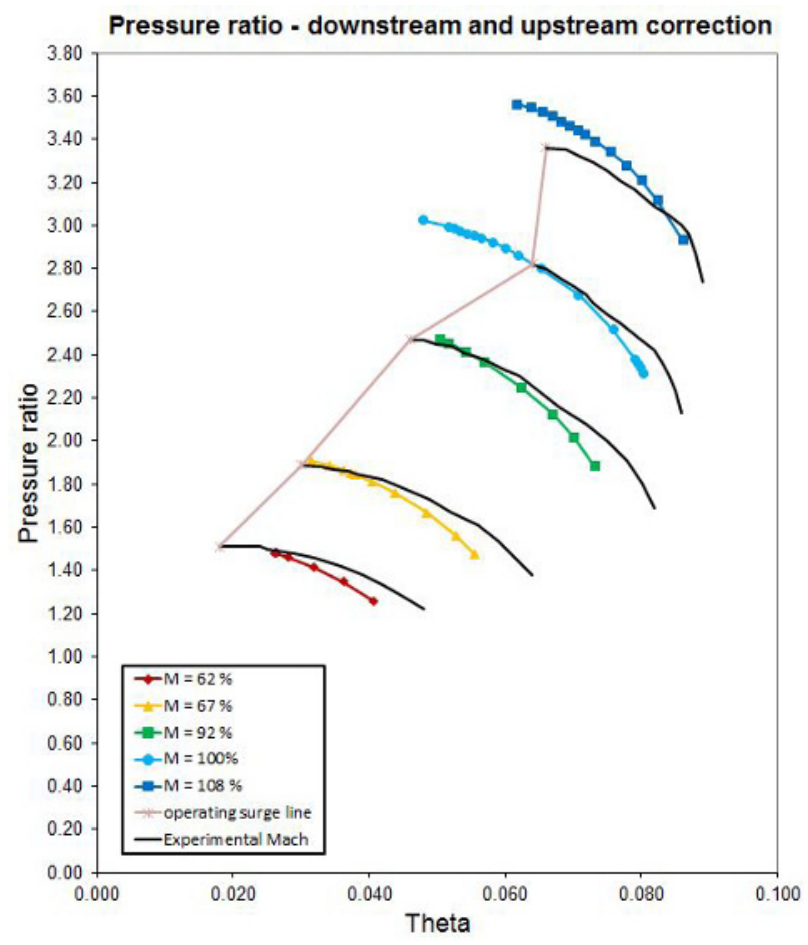

Fig. 10. Model composition correction - pressure ratio comparison

evolution is improvable in taking into account load losses for example.

We remark that the CFD curve slope was closer to the experimental line one before the correction.

Concerning the correction impact on efficiencies, it is almost insignificant, as we can see on the figure 11, given 
that we have considered isentropic evolutions. Curves have some differences with previous one because of the modification of computational points. Indeed, efficiencies are a bit better thanks to a lower pressure ratio, involving an efficiency increase for high rotational speed.

CFD efficiency values are always at least $2 \%$ higher than experiment.

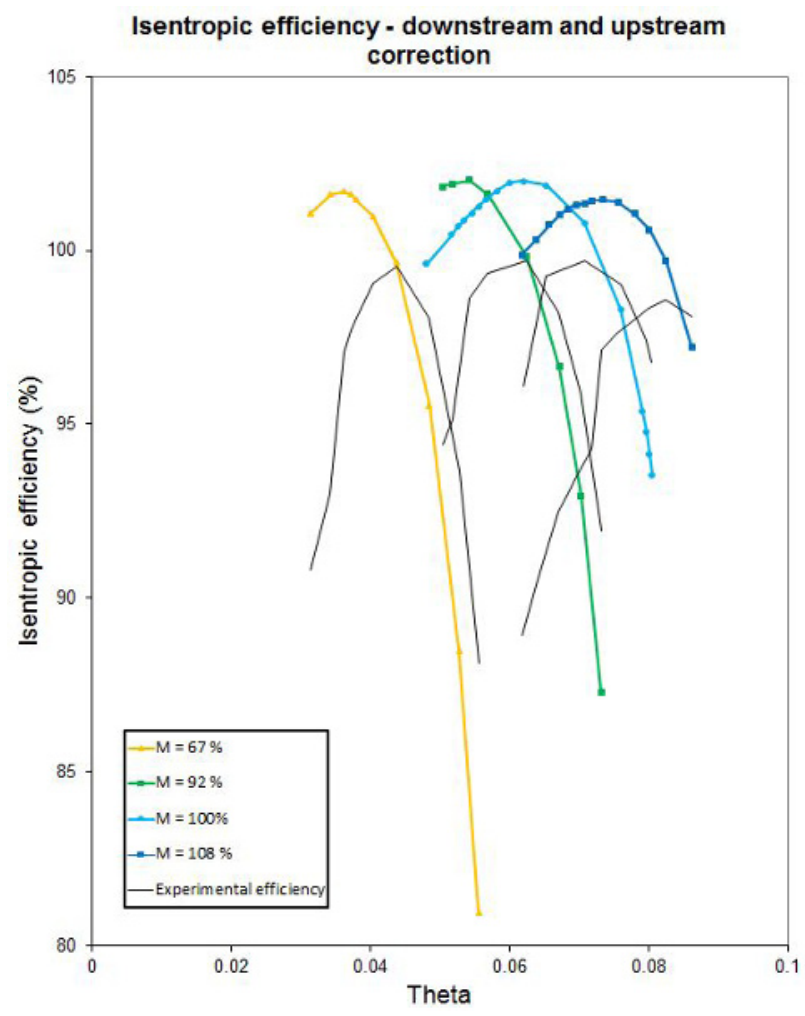

Fig. 11. Model composition correction - efficiency comparison

To improve gap between experimental and numerical values, we apply a second correction regarding the labyrinth seal leakages which are existing inside a compressor. The major one is a leakage between the impeller outlet and the compressor housing. This is due to the pressure gap existing between these two close areas.

The formula takes into account a mixing between the main flow at the impeller inlet with a secondary flow from the impeller outlet with higher temperature. Isentropic efficiency is dependant of temperature values as we can see on figure 13 .

CFD efficiency peaks are merged for Mach percentage equal to $92 \%$ and $100 \%$, and we have a good agreement for other curves. CFD flow rate are a bit lower than experiment results without exceeding acceptable values.

\subsection{Surge area analysis}

As we can see on all pressure ratio graphics, the $\mathrm{M}=100 \%$ CFD curve has many more points at low flow rate than the experimental line. This difference illustrates the gap between the operating surge limit and the real surge limit as



Fig. 12. Model composition correction + leak correction - pressure ratio comparison

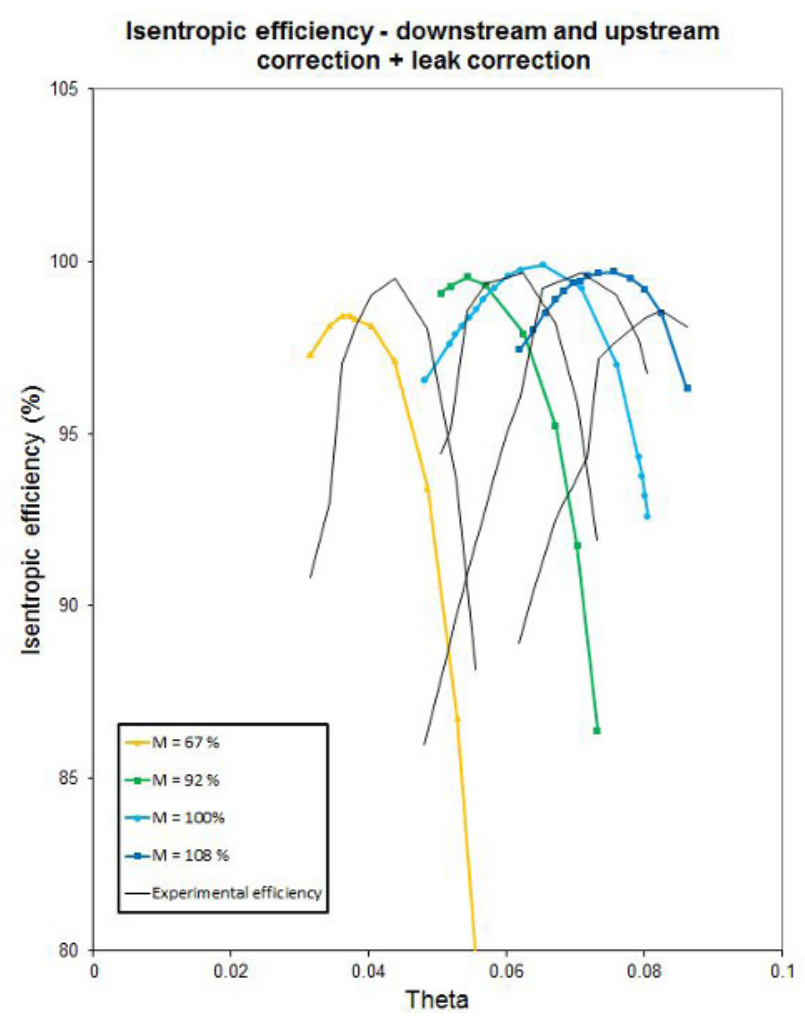

Fig. 13. Model composition correction + leak correction - efficiency comparison

shown on figure 1 .

The surge phenomenon is a transient appearance which may come from a flow separation in the impeller. CFD computations enable to characterize recirculations due to this flow separation. 




Fig. 14. Velocity vector field on a plane close to the impeller shroud - operating point at the efficiency peak

The figure 14 displays a velocity vector field on a plane close to the impeller shroud for an operating point at the efficiency peak.

We can observe that velocity vectors are rather homogeneous and do not let appear blue areas, indicative of low velocity zone.

Given that higher curvature is located on the impeller shroud, flow separation appears firstly in this impeller region as we can on the figure 15 .

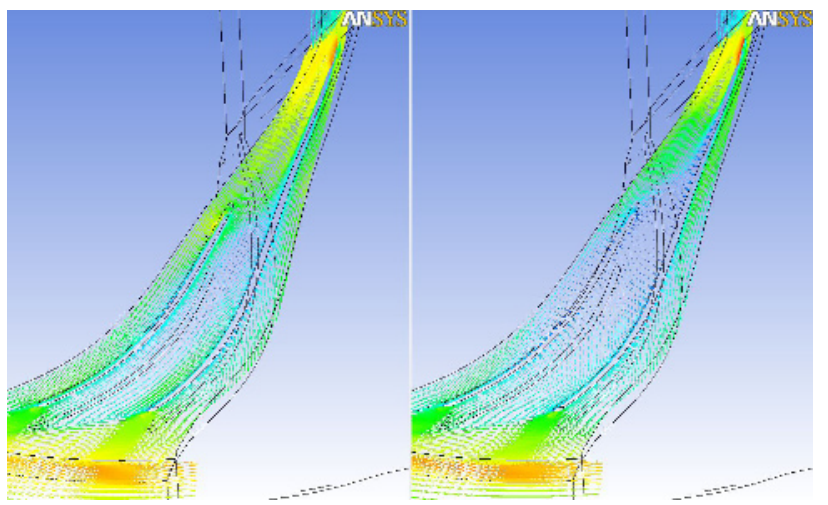

Fig. 15. Velocity vector field on a plane close to the impeller shroud - Left : operating surge point ; Right : CFD surge point

The left picture of this figure is a computation result close to the experimental operating surge limit. We can distinguish signs of velocity decrease at the beginning of the splitter (secondary blade).

The right picture is the last CFD point which has converged in decreasing flow rate. At this operating point, a large low velocity area is visible. In zooming in this flow separation, we note a recirculation thanks to the appearance of a vortex on the plane. Indeed, some vectors are directed towards the impeller inlet as we can see on the zoom, figure 16.

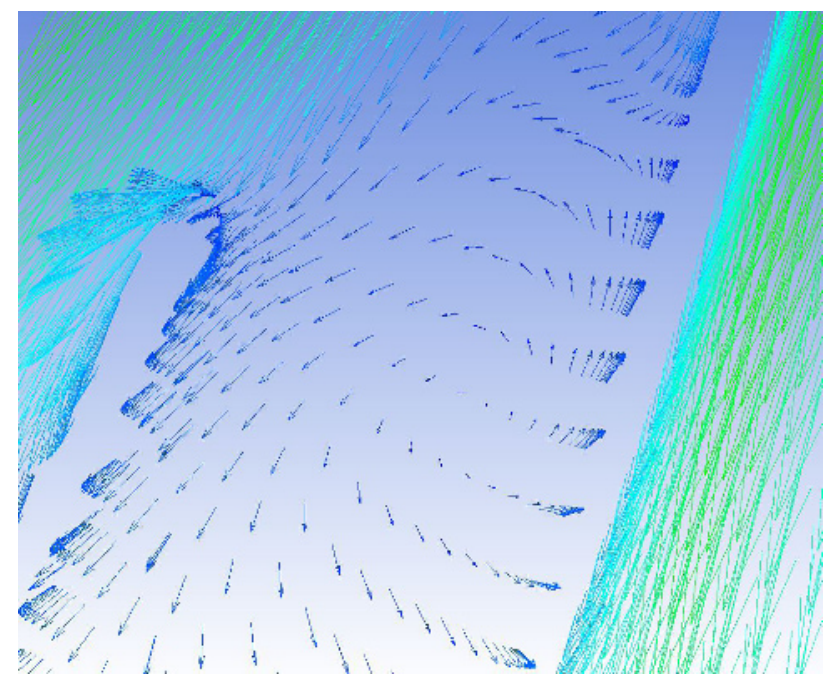

Fig. 16. Zoom in the recirculation area at surge point

We can suppose that this recirculation appearance is the source of surge. In trying to move this appearance forward lower flow rate, the operating range compressor could be get wider. But maybe to the detriment of high flow rate efficiency.

\section{Conclusion}

First computations have enabled to confirm that computational fluid dynamic is dependent of the mesh quality and may vary of some percent in function of the elements number.

An experimental rig precision depends also of the accuracy and the number of used sensors in it.

That is why it has been interesting to compare CFD results and verify that computations converged towards a value close to the experimental one.

Comparisons learn us that differences are varying with operating conditions, specially for high rotational velocities which involving higher pressure ratio and efficiency gradients.

Some corrections have contributed to improve CFD agreements with experimental values. However, our study shows these corrections may be made better because all curves are not merged yet. Moreover, corrections have some disadvantages like a tendency to modify speed line slope.

On the other hand, CFD enables a qualitative analysis which is particularly difficult with a test rig. It is possible to understand what is happening at surge approach and anticipate it within sight of getting a wider operating range. 


\section{References}

1. M. Hathaway, R. Chriss, J. Wood and A. Strazisar, NASA Technical Memorandum 4481, (1993)

2. B. Song, Experimental and numerical investigations of optimized high-turning supercritical compressor blades, Ph.D Thesis, (2003)

3. H. Pitkanen, H. Esa, P. Salinnen and J. Larjola, 4th int. symposium on experimental and computational aerothermodynamics of internal flows, (1999)

4. D. Roberts and R. Steed, ANSYS resource library, (2004)

5. Y. Galerkin, V. Mitrofanov and A. Prokofiev, International gas turbine congress, (2003)

6. S. Javeed and B. Reddy, Int. J. of fluids engeneering Vol. 3, No. 3, pages 335 to 342, (2011)

7. V. Mileshin, A. Startev and I. Orekhov, Proc. of the int. gas turbine congress, (2003)

8. M. Babak, Konference ANSYS, (2009)

9. M. Ding, C. Groth, S. Kacker and D. Roberts, ANSYS resource library, (2005)

10. N. Mostafa, A. Abdul-Gawad a,d M. Emeara, The Egyptian Int. J. of Eng. Sci and Technology, Vol. 14, No. 2, (2011)

11. Y. Iwakiri and H. Uchida, RED Review of Toyota $C R D L$, Vol. 41 No. 3, (2006)

12. S. Sivagnanasundaram, S. Spence and J. Early, 10th int. Conf on turbochargers and turbocharging, (2012)

13. J. Vierendeels and J. Degroote, Computational Fluid Dynamics, pages 305 to 328 , (2009)

14. G. Meauze, Technique de l'ingenieur, (1982) 